\title{
Propriedades biológicas de painéis de casca de arroz e adesivo tanino-formaldeído
}

\author{
Joel Telles de SOUZA ${ }^{1}$, Clóvis Roberto HASELEIN², Walmir Marques de MENEZES ${ }^{2}$, \\ Alencar GARLET ${ }^{3}$, Maiara TALGATTI ${ }^{2 *}$
}

\author{
${ }^{1}$ Universidade do Oeste de Santa Catarina, Xanxerê, SC, Brasil. \\ ${ }^{2}$ Universidade Federal de Santa Maria, Santa Maria, RN, Brasil. \\ ${ }^{3}$ Serviço Florestal Brasileiro, Brasília, DF, Brasil. \\ *E-mail: maiara.talgatti@gmail.com
}

Recebido em setembro/2017; Aceito em março/2018.

\begin{abstract}
RESUMO: O presente estudo tem como objetivo avaliar as propriedades biológicas, por meio de ensaio de apodrecimento acelerado com fungos xilófagos, em chapas aglomeradas de casca de arroz (Oryza sativa $\mathrm{L}$ ) coladas com adesivo à base de tanino-formaldeído. Para tanto, foram utilizadas casca de arroz na forma natural e processada em moinho de martelo, compactadas a diferentes massas específicas $\left(0,65 ; 0,95\right.$ e $\left.1,15 \mathrm{~g} . \mathrm{cm}^{-3}\right)$ e aplicando-se teores de adesivos de 7, 10 e 13\%, totalizando 54 chapas. Os resultados de perda de massa foram submetidos ao teste de correlação simples de Pearson e analisados por regressão. De acordo com os requisitos da norma ASTM D-2017 (2005), os testes biológicos, para todos os tratamentos, foram classificados como resistentes ao fungo Trametes versicolor, podridão branca. Já para o fungo Gloeophyllum trabeum a maioria dos tratamentos foram classificados como moderadamente resistente. Portanto, a casca de arroz mostra-se como uma alternativa de elevado potencial para a manufatura de chapas de maior resistência aos fungos apodrecedores de madeiras.
\end{abstract}

Palavras-chave: massa específica, correlação simples de Pearson, fungos apodrecedores.

\section{Biological properties of rice husk particleboard manufactured with tannin-formaldehyde}

\begin{abstract}
Resistance to decay fungi Trametes versicolor and Gloephyllum trabeum of panels produced with rice husk and tannin - formaldehyde adhesive was evaluated. In the production of the panels, rice hulls were used in its natural form or hammer milled, compacted at different densities $\left(0.65,0.95\right.$ and $\left.1.15 \mathrm{~g} / \mathrm{cm}^{3}\right)$ and with adhesives levels of 7, 10 and $13 \%$ (based on the ovendry weight of the particles. The results were submitted to the Pearson correlation test and analyzed by regression. According to the requirements of ASTM D- 2017 (2005), the biological tests for all treatments were classified as resistant to white rot ungus Trametes versicolor. For the brown rot fungus Gloeophyllum trabeum, most treatments were classified as moderately resistant. Therefore, rice husk can be a high potential alternative to for the manufacture of particleboards for greater resistance to decay wood fungi.
\end{abstract}

Keywords: specific gravity, simple Pearson correlation, decay fungi.

\section{INTRODUÇÃO}

As chapas aglomeradas podem ser fabricadas a partir de qualquer material lignocelulósico desde que thes confira resistência mecânica adequada. Estes materiais supracitados utilizados como base para a formação de painéis são advindos de madeira de florestas ou reflorestamentos e também de aproveitamento de resíduos industriais florestais ou da agricultura. No Brasil, as matérias-primas utilizadas na confecção de chapas são basicamente madeiras de Pinus sp. e Eucalyptus sp. (IWAKIRI et al., 2010). Baldin et al., 2016, destacam que esses materiais são os preferidos devido ao crescimento rápido, à rotação curta e às altas taxas de retorno. Porém, painéis à base de madeira e outros materiais lignocelulósicos são susceptíveis aos organismos xilófagos (MELO et al., 2010).

A indústria de painéis apresenta suma importância para a economia brasileira, principalmente pela geração de novas tecnologias e emprego nos setores moveleiro e da construção civil (Vieira et al., 2012). Nos últimos anos, a tendência de utilização de resíduos pela indústria nacional tem despertado o interesse de vários autores como: Stangerling et al., 2011; Baldin et al., 2016 e Machado et. al., 2017. Um material antes considerado resíduo, passa a ser tratado como um subproduto do processo produtivo por meio da sua valorização como matéria-prima na fabricação de novos produtos (TALGATTI et al. 2017).

De acordo com Riceboard (2016), o desenvolvimento da tecnologia para manufatura de chapas aglomeradas, utilizando casca de arroz, destaca-se como uma das melhores soluções para os problemas dos rejeitos da agricultura e, ao mesmo tempo, da preservação das florestas. Estas chapas podem substituir os aglomerados de partículas de madeira em diversos tipos de uso.

O arroz está entre os cereais mais consumidos no mundo, seu cultivo ocupa o segundo lugar em área plantada no mundo, só perdendo para o trigo. $\mathrm{O}$ maior produtor é a China, seguido da Índia, Indonésia, Bangladesh, Vietnã e outros (MINISTÉRIO DA AGRICULTURA, 2015).

Em 2005 o Brasil ocupava o décimo lugar, produzindo cerca de $2 \%$ da produção mundial (Associação Brasileira das Indústrias de Arroz Parboilizado-ABIAP, 2005), o país produzia cerca de 12 milhões de toneladas de arroz com casca anualmente. Entre os estados brasileiros, o Rio Grande do Sul era o maior produtor, com uma área plantada de, 
aproximadamente, um milhão de hectares (Ministério da Agricultura, 2015). Ainda de acordo com o Ministério da Agricultura (2015), o Brasil é o nono maior produtor mundial e colheu 11,26 milhões de toneladas na safra 2009/2010, em uma área de cultivo menor.

Neste contexto, o presente estudo tem como objetivo avaliar as propriedades biológicas, por meio de ensaio de apodrecimento acelerado com fungos xilófagos, em chapas aglomeradas de casca de arroz (Oryza sativa L) coladas com adesivo à base de tanino-formaldeído.

\section{MATERIAL E MÉTODOS}

A casca de arroz utilizada nesta pesquisa foi submetida ao processo de preparação, que consistiu em passagem por moinho de martelo (partículas processadas) e não processada (partículas naturais). Posteriormente, as partículas foram secas em estufa, a aproximadamente, $50^{\circ} \mathrm{C}$ por um período que variou entre 12 a 24 horas, até o teor de umidade de $3 \%$, ideal para confecção das chapas.

$\mathrm{O}$ adesivo foi produzido em laboratório, com o tanino da acácia-negra (Acacia mearnsii De Wild), na forma de pó, hidratado 24 horas antes da formulação, por meio do preparo de uma solução em água com aproximadamente $50 \%$ de sólidos. Após, foi adicionado hidróxido de sódio $(\mathrm{NaOH})$ até que o pH 8 fosse atingido, sendo que, para esta medição, utilizou-se de pHmetro. $\mathrm{O}$ formaldeído foi adicionado apenas no momento da aplicação do adesivo, sendo utilizado na proporção de $10 \%$ (base peso seco do tanino).

Para o monitoramento do teor de umidade das partículas de casca de arroz, após a secagem, foram separadas 5 amostras que foram secas a $103^{\circ} \mathrm{C}$, até massa constante. Quando necessário, foi adicionado certa quantidade de água para que o material atingisse a umidade estabelecida de 3\%. Em seguida, as partículas foram então misturadas com o adesivo taninoformaldeído. As chapas foram confeccionadas com dimensões de $40 \mathrm{~cm}$ x $40 \mathrm{~cm}$ x $0,95 \mathrm{~cm}$, com pressão específica de 30 $\mathrm{kgf} / \mathrm{cm}^{2}$ e temperatura dos pratos ajustada para $180^{\circ} \mathrm{C}$. A espessura de $0,95 \mathrm{~cm}$ foi definida por gabaritos de barras de aço, as quais foram colocadas nas laterais do colchão, limitando o fechamento da prensa. $\mathrm{O}$ tempo total de prensagem aplicado para promover a evaporação da água e a cura do adesivo foi de 8 minutos. Após a prensagem, as chapas foram identificadas, lixadas e tiveram suas bordas aparadas, passando para as dimensões finais de $39 \mathrm{~cm} \times 39 \mathrm{~cm} \times 0,95$ $\mathrm{cm}$. Estas foram dispostas em câmara climatizada $\left(20^{\circ} \mathrm{C}\right.$ e $65 \%$ de umidade relativa) até massa constante.

$\mathrm{O}$ ensaio de apodrecimento acelerado foi conduzido no setor de Biodegradação e Preservação da Madeira, do Laboratório de Produtos Florestais (LPF) do Serviço Florestal Brasileiro, em Brasília, DF. A metodologia empregada foi a proposta pela norma da American Society for Testing and Materials- ASTM D-2017 (2005). As amostras foram preparadas nas dimensões de $2,5 \mathrm{~cm}$ x $2,5 \mathrm{~cm}$, e espessura igual à da chapa. Após foram enviadas ao Laboratório de Produtos Florestais-LPF do Serviço Florestal Brasileiro, para ensaio de apodrecimento acelerado.

Todos os corpos de prova foram numerados com caneta de escrita permanente, identificando o tratamento e número da amostra. O material foi acondicionado em estufa com circulação forçada de ar a $50^{\circ} \mathrm{C}$, até atingir massa constante. Posteriormente, foram pesados em balança analítica com precisão de $0,0001 \mathrm{~g}$ e o resultado registrado como massa inicial (Mi).

Para cada tratamento foram ensaiados 20 corpos de prova, sendo 8 para podridão branca, 6 para podridão parda e 6 blocos de correção (sem fungo). Após a pesagem inicial, os corpos de prova foram esterilizados em autoclave, a $121^{\circ} \mathrm{C}$ por 45 minutos. Foram empregadas dois representantes de fungos xilófagos provenientes da coleção do LPF: Trametes versicolor (Linnaeus ex Fries) Pilat, causador de podridão branca, e Gloeophyllum trabeum (Persoon. ex Fries.) Murr. causador de podridão parda. Para cada fungo foram preparadas 10 placas de petri com condições ideais para 0 desenvolvimento dos fungos. Após, as placas foram levadas à incubadora por duas semanas para o crescimento do micélio. Na sequência, já em cultura em meio líquido, foram utilizados frascos erlenmeyer de $1000 \mathrm{~mL}$ de capacidade total, contendo $200 \mathrm{~mL}$ de meio malte líquido a 3\%. Foram preparados quatro frascos para cada espécie de fungo.

Posteriormente a colonização das placas suporte, introduziu-se um corpo de prova em cada frasco de ensaio, os quais permaneceram na incubadora por 14 semanas em contato com os fungos com temperatura ajustada para $26( \pm 1){ }^{\circ} \mathrm{C}$ e umidade relativa de $70( \pm 5) \%$.

Foram utilizados 16 corpos de prova $(2,5 \mathrm{~cm} \times 2,5 \mathrm{~cm} \mathrm{x}$ $0,9 \mathrm{~cm}$ ) de pinus (Pinus sp.) como espécie de referência para o fungo Gloeophyllum trabeum e 16 de embaúba (Cecropia sp.) para o fungo Trametes versicolor. Os corpos de prova de referência são indicadores do vigor vegetativo da cultura fúngica utilizada no ensaio e das condições de incubação. Segundo a norma ASTM D 2017 (2005), o teste é considerado válido somente quando o fungo provoca perda de massa de $50 \%$ sobre as amostras de referência, num período de até 16 semanas. A partir dos valores de massa inicial e final, foi possível calcular a perda de massa individual de cada corpo de prova e, posteriormente, a média de cada tratamento. De posse destes dados, os tratamentos foram classificados quanto à resistência ao ataque dos fungos, segundo os critérios estabelecidos pela ASTM D 2017 (2005), apresentado na Tabela 1.

Tabela 1. Classes de resistência ao apodrecimento com fungos xilófagos, segundo a norma ASTM D 2017 (2005).

Table 1. Decay resistance of wood according to ASTM D 2017 (2005) standard.

\begin{tabular}{lcc}
\hline \multicolumn{1}{c}{ Classes de Resistência } & $\begin{array}{c}\text { Perda de } \\
\text { Massa (\%) }\end{array}$ & $\begin{array}{c}\text { Massa } \\
\text { Residual (\%) }\end{array}$ \\
\hline Altamente Resistente (AR) & $0-10$ & $90-100$ \\
Resistente (R) & $11-24$ & $76-89$ \\
Moderadamente Resistente (MR) & $25-44$ & $56-75$ \\
Não-Resistente (NR) & $>45$ & $>55$ \\
\hline
\end{tabular}

O experimento foi realizado com as seguintes avaliações: teor de adesivos (TA): 7, 10 e 13\% (com base no peso seco das partículas); massa específica (ME) das chapas: 0,65, 0,95 e $1,15 \mathrm{~g} . \mathrm{cm}^{-3}$; preparo das partículas: Natural (N) e Processada (P) em moinho de martelo.

Estas combinações perfizeram um total de 18 tratamentos, sendo que, para cada um destes foram realizadas 3 repetições, totalizando, portanto, 54 chapas confeccionadas a partir do resíduo de casca de arroz. Os dados foram processados e analisados utilizando-se o programa Excel ${ }^{\circledR}$ 2007, e de um 
programa de análise específico para atender os objetivos da pesquisa, o software Statgraphics Centurion.

Os resultados dos ensaios biológicos foram submetidos à análise de correlação simples de Pearson a $5 \%$ de probabilidade de erro, utilizando-se as variáveis de massa específica observada (Meo) e teor de adesivo (TA). Após, os dados foram analisados pelo método Stepwise e por regressão linear múltipla a $5 \%$ de probabilidade de erro.

\section{RESULTADOS}

Na Tabela 2, é possível a visualização dos valores médios de perda de massa para cada tratamento, resultante dos ensaios de apodrecimento acelerado com fungos xilófagos do tipo podridão branca (Trametes versicolor) e podridão parda (Gloeophyllum trabeum).

Os ensaios biológicos tiveram valores de massa específica observada bem próxima às massas específicas nominais de $0,65,0,95$ e $1,15 \mathrm{~g}_{\mathrm{cm}}{ }^{-3}$. A perda de massa observada nas madeiras de referência foi de $54,90 \%$ a espécie de Cecropia sp., em contato com o fungo Trametes versicolor, e de $62,19 \%$ na madeira de Pinus sp., ocasionada pelo Gloeophyllum trabeum.

Na Tabela 3, verifica-se a matriz de correlação entre a perda de massa para podridão branca $(\mathrm{PB})$ e parda $(\mathrm{PP})$ e as propriedade de TA e Meo dos corpos de prova. A única correlação a $5 \%$ de probabilidade de erro, para chapas de partículas naturais foi entre PP e Meo $(-0,46)$, sendo que não houve essa tendência para as demais propriedades. Nas chapas de partículas processadas, foi observado correlação entre PP e MEo $(-0,54)$ e PP e TA $(-0,28)$, não havendo tal comportamento para as demais propriedades. Assim, a melhor correlação foi entre PP e Meo $(-0,54)$ em painéis de partículas processadas. Além disso, a perda de massa para $\mathrm{PB}$ não teve correlação com nenhuma das propriedades contidas nas chapas, para ambos os tipos de partículas.

A análise de regressão não constatou diferença entre a perda de massa para Trametes versicolor (PB), ME e TA, tanto para partículas naturais quanto processadas tendência que também foi verificado na Matriz de correlação simples de Pearson, na Tabela 5, reforçando, portanto, o resultado encontrado, não houve uma tendência de acréscimo ou decréscimo da resistência da chapa à perda de massa.

Entretanto, a perda de massa para o Gloeophyllum trabeum (PP) apresentou diferença com o acréscimo da ME. Na Figura 1 , nota-se que à medida que aumenta a $\mathrm{ME}$, ocorre maior resistência da chapa ao ataque do fungo causador da podridão parda, já que ocorre a diminuição da perda de massa. Este comportamento é verificado nos dois tipos de partículas utilizadas no presente estudo.

Tabela 2. Tratamentos, teor de adesivos (TA), partículas naturais $(\mathrm{N})$ e processada (P), massa específica nominal (Men) e observada (Meo) e valores médios de perda de massa (PM) para os fungos Trametes versicolor e Gloeophyllum trabeum.

Table 2. Treatments, adhesive content (TA), natural (N) and processed (P) particles, nominal (Men) and observed density (Meo) and average values of loss of mass (PM) for Trametes versicolor and Gloeophyllum trabeum fungus.

\begin{tabular}{|c|c|c|c|c|c|c|}
\hline Trat. & $\begin{array}{l}\text { TA } \\
(\%)\end{array}$ & $\begin{array}{l}\text { Men } \\
\left(\text { g.cm }^{-3}\right)\end{array}$ & $\begin{array}{r}\text { Partículas } \\
(\mathrm{N}, \mathrm{P})\end{array}$ & $\begin{array}{l}\text { Meo } \\
\left(\text { g.cm } \mathrm{cm}^{-3}\right)\end{array}$ & $\begin{array}{l}\text { T. versicolor } \\
\text { PM }(\%)\end{array}$ & $\begin{array}{l}\text { G. trabeum } \\
\text { PM }(\%)\end{array}$ \\
\hline $\mathrm{T} 1$ & \multirow{6}{*}{7} & \multirow{2}{*}{0,65} & $\mathrm{~N}$ & 0,630 & $18,79 \mathrm{R}$ & $28,10 \mathrm{MR}$ \\
\hline $\mathrm{T} 2$ & & & $\mathrm{P}$ & 0,639 & $16,69 \mathrm{R}$ & $30,68 \mathrm{MR}$ \\
\hline $\mathrm{T} 3$ & & \multirow{2}{*}{0,95} & $\mathrm{~N}$ & 0,930 & $17,28 \mathrm{R}$ & $29,59 \mathrm{MR}$ \\
\hline $\mathrm{T} 4$ & & & $\mathrm{P}$ & 0,930 & $18,40 \mathrm{R}$ & 29,69 MR \\
\hline $\mathrm{T} 5$ & & \multirow{2}{*}{1,15} & $\mathrm{~N}$ & 1,121 & $16,04 \mathrm{R}$ & $27,67 \mathrm{MR}$ \\
\hline $\mathrm{T} 6$ & & & $\mathrm{P}$ & 1,125 & $16,69 \mathrm{R}$ & $26,11 \mathrm{MR}$ \\
\hline $\mathrm{T} 7$ & \multirow{6}{*}{10} & \multirow{2}{*}{0,65} & $\mathrm{~N}$ & 0,636 & $17,27 \mathrm{R}$ & $31,95 \mathrm{MR}$ \\
\hline $\mathrm{T} 8$ & & & $\mathrm{P}$ & 0,638 & $20,11 \mathrm{R}$ & $29,95 \mathrm{MR}$ \\
\hline T9 & & \multirow{2}{*}{0,95} & $\mathrm{~N}$ & 0,935 & $19,18 \mathrm{R}$ & $28,41 \mathrm{MR}$ \\
\hline $\mathrm{T} 10$ & & & $\mathrm{P}$ & 0,928 & $20,44 \mathrm{R}$ & $25,07 \mathrm{MR}$ \\
\hline $\mathrm{T} 11$ & & \multirow{2}{*}{1,15} & $\mathrm{~N}$ & 1,129 & $13,31 \mathrm{R}$ & $19,49 \mathrm{R}$ \\
\hline $\mathrm{T} 12$ & & & $\mathrm{P}$ & 1,129 & $18,04 \mathrm{R}$ & $23,65 \mathrm{R}$ \\
\hline T13 & \multirow{6}{*}{13} & \multirow{2}{*}{0,65} & $\mathrm{~N}$ & 0,636 & $17,30 \mathrm{R}$ & $29,78 \mathrm{MR}$ \\
\hline $\mathrm{T} 14$ & & & $\mathrm{P}$ & 0,634 & $19,15 \mathrm{R}$ & $30,65 \mathrm{MR}$ \\
\hline $\mathrm{T} 15$ & & \multirow{2}{*}{0,95} & $\mathrm{~N}$ & 0,930 & $16,62 \mathrm{R}$ & $25,34 \mathrm{MR}$ \\
\hline $\mathrm{T} 16$ & & & $\mathrm{P}$ & 0,931 & $17,85 \mathrm{R}$ & $23,51 \mathrm{R}$ \\
\hline $\mathrm{T} 17$ & & \multirow{2}{*}{1,15} & $\mathrm{~N}$ & 1,131 & $16,32 \mathrm{R}$ & $24,90 \mathrm{R}$ \\
\hline $\mathrm{T} 18$ & & & $\mathrm{P}$ & 1,130 & $16,18 \mathrm{R}$ & $22,02 \mathrm{R}$ \\
\hline \multicolumn{6}{|c|}{ Pinus sp. (referência para podridão parda) } & $62,19 \mathrm{NR}$ \\
\hline \multicolumn{5}{|c|}{ Cecropia sp. (referência para podridão branca) } & $54,90 \mathrm{NR}$ & \\
\hline
\end{tabular}

Tabela 3. Matriz de correlação simples de Pearson para a perda de massa através do ensaio de apodrecimento acelerado com fungos xilófagos. Table 3. Pearson correlation coefficient for loss of mass through the accelerated test with wood decay-producing fungi.

\begin{tabular}{|c|c|c|c|c|c|c|c|c|}
\hline \multicolumn{3}{|c|}{ Partícula Natural } & \multicolumn{6}{|c|}{ Partícula Processada } \\
\hline & Meo & TA & $\mathrm{PB}$ & $\mathrm{PP}$ & Meo & TA & $\mathrm{PB}$ & $\mathrm{PP}$ \\
\hline Meo & 1 & & & & 1 & & & \\
\hline TA & $0,01 \mathrm{~ns}$ & 1 & & & $0,002 \mathrm{~ns}$ & 1 & & \\
\hline PB & $-0,26 \mathrm{~ns}$ & $-0,07 \mathrm{~ns}$ & 1 & & $-0,19 \mathrm{~ns}$ & $0,19 \mathrm{~ns}$ & 1 & \\
\hline PP & $-0,46 \mathrm{~s}$ & $-0,14 \mathrm{~ns}$ & $0,20 \mathrm{~ns}$ & 1 & $-0,54 \mathrm{~s}$ & $-0,28 \mathrm{~s}$ & $0,15 \mathrm{~ns}$ & 1 \\
\hline
\end{tabular}

Meo = Massa Específica observada $\left(\mathrm{g} . \mathrm{cm}^{-3}\right) ; \mathrm{TA}=$ Teor de adesivo (\%); $\mathrm{PB}=$ podridão branca através do fungo Trametes versicolor $(\%) ;$ PP $=$ podridão parda através do fungo Gloeophyllum trabeum $(\%)$; s e ns = significativo e não significativo a $5 \%$ de probabilidade de erro pelo teste de correlação de Pearson. 

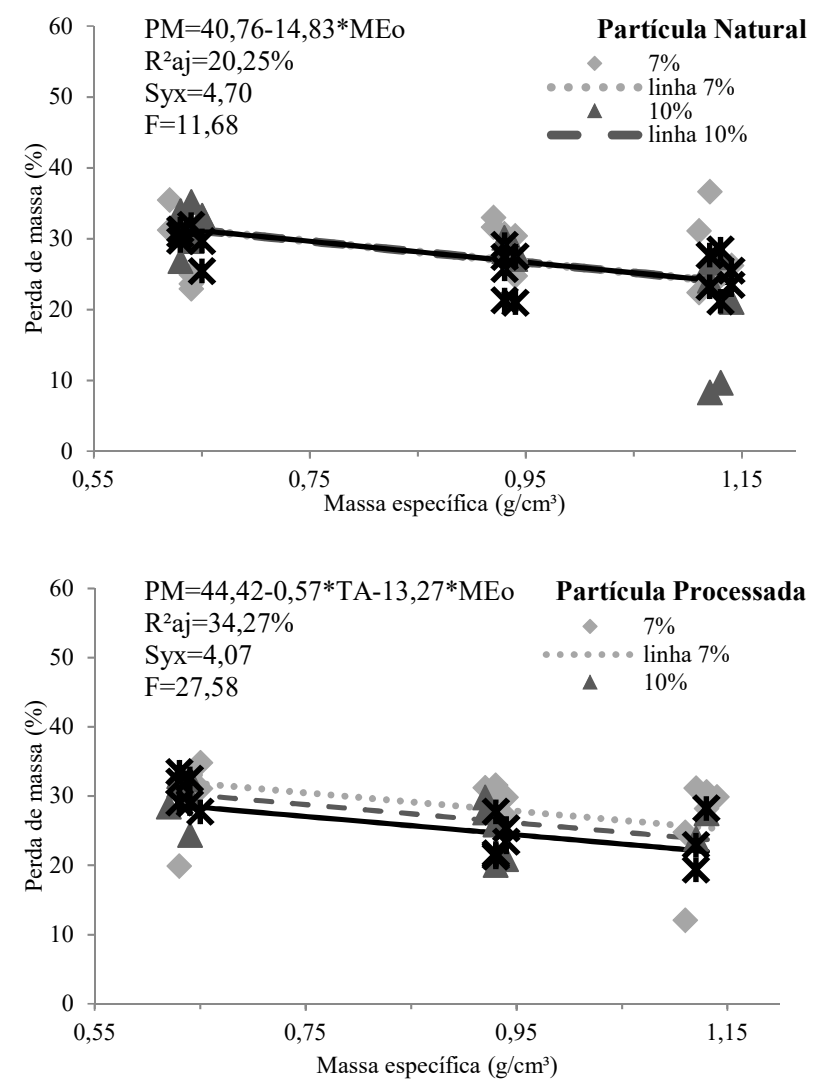

Figura 1. Valores de perda de massa observados e ajustados para o fungo Gloeophyllum trabeum, em função da massa específica, para chapas de partículas naturais e processadas, com 3 teores de adesivos. Figure 1. Loss of mass values observed and adjusted for the Gloeophyllum trabeum fungi, as a function of density, for natural and processed particleboards, with three adhesive levels.

Quanto ao TA, também foi constatado diferença, para as chapas confeccionadas com partículas processadas. Com o aumento do adesivo tanino-formaldeído, ocorreu o acréscimo da resistência ao ataque do fungo Gloeophyllum trabeum, causador da podridão parda. Já para as chapas de partículas naturais não foi observado diferença de perda de massa com o aumento do TA.

\section{DISCUSSÃO}

Os valores de perda de massa foram observados após 14 semanas de ensaio e são superiores ao valor mínimo de 50\% estabelecido pela norma ASTM D 2017 (2005). Estes dados atestam que as condições gerais de ensaios foram favoráveis ao desenvolvimento dos fungos, e que as culturas empregadas estavam em pleno vigor vegetativo, portanto, os resultados do ensaio são válidos.

De acordo com os critérios da norma americana, ASTM D 2017 (2005), todos os tratamentos foram classificados como resistentes ao fungo Trametes versicolor para podridão branca. Isto indica que a resistência ao apodrecimento foi pouco afetada pelas variáveis testadas, sendo devido, principalmente, à resistência intrínseca da casca de arroz. Em estudos com painéis aglomerados confeccionados com Eucalyptus grandis e Bambusa vulgaris em ensaio de apodrecimento acelerado, Stagerling et al. (2011), não obtiveram resultados satisfatórios e todas as formulações empregadas foram classificadas como moderadas.
Para o fungo Gloeophyllum trabeum (podridão parda), a maioria dos tratamentos foi classificada como moderadamente resistente. Para este fungo, o aumento da densidade da chapa e do teor de adesivo diminuiu a perda de massa, sendo os tratamentos T11, T12, T16, T17 e T18 classificados como resistentes ao apodrecimento. A influência da ME e do TA sobre a resistência biológica pode ser visualizada na Figura 1.

Okino et al. (2007), ao estudarem a resistência de chapas OSB de Pinus taeda, relatam que o aumento do TA proporcionou maior resistência ao fungo apodrecedor.

Os tratamentos classificados como resistentes podem ser utilizados em situações onde haja risco de umidificação eventual, porém fora do contado com solo ou água. Materiais classificados como moderadamente resistentes só devem ser usados fora do contato com a umidade, sob pena de serem atacados por fungos. Para estes aglomerados são necessários estudos da aplicação de produtos preservativos para melhorar sua resistência ao ataque de fungos xilófagos (DIROL; DEGLISE, 2001).

Entretanto, para fins de indicação de uso das chapas, por razões de segurança, deve-se considerar a pior situação. Como não é possível prever qual espécie de fungo irá atacar as peças em serviço, devem-se considerar os dados do fungo que apresentou a maior perda de massa, como indicador da durabilidade do material avaliado.

Considerando o exposto acima, as chapas que podem ser utilizadas em ambientes de umidade eventual são as de tratamentos T11, T12, T16, T17 e T18, classificados como resistentes ao apodrecimento para ambos os tipos de fungos analisados neste estudo.

Ademais, Stangerling et al. (2011) mencionam que a classificação "moderadamente resistente", indicada pela ASTM D 2017 (2005), e obtida por parte dos tratamentos em estudo para podridão parda, não inviabiliza o uso das chapas, contanto que estas não venham a ser utilizadas em ambientes adversos, tais como: uso exterior e em contato direto com o solo.

\section{CONCLUSÕES}

De acordo com os critérios da norma americana, ASTM D 2017 (2005), todos os tratamentos foram classificados como resistentes ao fungo Trametes versicolor para podridão branca. Isto indica que a resistência ao apodrecimento foi pouco afetada pelas variáveis testadas devido, principalmente, à resistência intrínseca da casca de arroz. Para o fungo Gloeophyllum trabeum (podridão parda), a maioria dos tratamentos foram classificados como moderadamente resistente. De modo geral, a utilização de casca de arroz revelou ser uma alternativa de elevado potencial para a confecção de chapas de maior resistência a fungos apodrecedores.

\section{REFERÊNCIAS}

BARBOZA, R. I.; FEARNSIDE, P.M. Incêndios na Amazônia brasileira: estimativa da emissão de gases do efeito estufa pela queima de diferentes ecossistemas de Roraima na paisagem do evento "El Niño" (1997/98). Acta Amazônica, Manaus, v. 29, p. 513-534, 1999.

ASSOCIAÇÃO BRASILEIRA DAS INDÚSTRIAS DE ARROZ PARBOILIZADO- ABIAP, 2005. Diponível em: http://abiap.com.br. 
AMERICAN SOCIETY FOR TESTING AND MATERIALS

- ASTM D 2017: Standard method for accelerated laboratory test of natural decay resistance of woods. Annual Book of ASTM Standards, Philadelphia. v.0410, p.5, 2005.

BALDIN, T.; SILVEIRA, A. G.; VIDRANO, B. R. A.; CANCIAN, L. C.; SPATT, L. L.; HASELEIN, C. R. Qualidade de painéis aglomerados produzidos com diferentes proporções de madeira e capim-annoni. Revista Brasileira de Ciências Agrárias, Recife, v. 11, n. 3, p. 230-237, 2016.

DOI: http://dx.doi.org/10.5039/agraria.v11i3a5376

DIROL, D.; DEGLISE, X. Durabilitè dês bois et problèmes associés. Paris: Hermes Science Europe Ltd, p. 416, 2001.

IWAKIRI, S.; ZELLER, F.; PINTO, J. A.; RAMIREZ, M.G.; SOUZA, M. M.; SEIXAS, R. Avaliação do potencial de utilização da madeira de Schizolobium amazonicum "Paricá" e Cecropia hololeuca "Embaúba" para produção de painéis aglomerados. Acta Amazonica, Manaus, v. 40, n. 2, p. 303-308, 2010.

MINISTÉRIO DA AGRICULTURA. 2015. Disponível em: http://agricultura.gov.br

MACHADO, N. A. F.; FURTADO, M. B.; PARRASERRANO, L. J.; PARENTE, M. O. M.; SAVASTANO JÚNIOR, J. F. H. Painéis aglomerados fabricados com resíduos do coco babaçu. Revista Brasileira de Ciências Agrárias, Recife, v. 12, n. 2, p. 202-209, 2017. DOI: http://dx.doi.org/10.5039/agraria.v12i2a5434

MELO, R. R.; SANTINI, E. J.; HASELEIN, C. R.; GARLET, A.; PAES, J. B.; STANGERLIN, D. M. Resistência de painéis aglomerados produzidos com diferentes proporções de madeira e casca de arroz a fungos e cupins xilófagos. Ciência Florestal, Santa Maria, v. 20, n. 3, p. 501-511, 2010 .

http://dx.doi.org/10.5902/198050982064
OKINO, E. Y. A. Biodegradação de chapas de partículas orientadas de pinus, eucalipto e cipreste expostas a quatro fungos apodrecedores. Scientia Forestalis, Piracicaba, v. 74, p. 67-74, 2007.

RICEBORARD. Technology. offer. doc. Disponível em:< http://www.nrdcindia.com/pages/ricebord.htm,2007>. Aceso em: jan. de 2016.

SANTANA, M. A. E.; TEIXEIRA, D. E. Uso de bagaço de cana-de-açúcar na confecção de chapas aglomeradas. Brasília: IBAMA; 1996.

STANGERLIN, D. M; MELO, R. R.; GARLET, A.; GATTO, D. A. Durabilidade natural de painéis aglomerados confeccionados com Eucalyptus grandis e Bambusa vulgaris em ensaio de apodrecimento acelerado. Ciência Rural, Santa Maria, v. 41, n. 8, p. 1369- 1374, 2011. DOI: http://dx.doi.org/10.1590/S0103-84782011000800012

TALGATTI, M.; BALDIN, T.; SILVEIRA, A. G.; SANTINI, E.J.; VIDRANO, B.R.A. Compósito madeira-plástico a partir de resíduos de três espécies florestais. Pesquisa Florestal Brasileira, Colombo, v. 37, n. 91, p. 277-283, 2017.

DOI: http://dx.doi.org/10.4336/2017.pfb.37.91.1385

VIEIRA, M. C.; BRITO, E. O.; GONÇALVES, F. G. Evolução econômica do painel compensado no Brasil e no mundo. Floresta e Ambiente, Seropédica, n. 19, v. 3, p. 277-285, 2012.

DOI: 